\title{
Stability during in vitro digestion of lactoferrin-loaded liposomes prepared from milk fat globule membrane-derived phospholipids
}

\author{
Weilin Liu, ${ }^{*} \dagger$ Aiqian Ye, $\dagger^{1}$ Wei Liu, ${ }^{*}$ Chengmei Liu, ${ }^{* 1}$ and Harjinder Singh $\dagger$ \\ *State Key Laboratory of Food Science and Technology, Nanchang University, No. 235, Nanjing East Road, Nanchang, 330047, \\ Jiangxi, P.R. China \\ †Riddet Institute, Massey University, Private Bag 11 222, Palmerston North 4442, New Zealand
}

\begin{abstract}
Liposomes loaded with positively charged lactoferrin (LF) were prepared from milk fat globule membrane-derived phospholipids using a thin-layer dispersion method. The entrapment efficiency of LF in the liposomes and the stability during in vitro gastrointestinal digestion were characterized and examined using dynamic light scattering, transmission electron microscopy, and PAGE. The entrapment efficiency of LF encapsulated in the liposomes was about $46 \%$. The entrapped LF remained unchanged as a function of time and pepsin concentration when the liposome samples were digested in a simulated gastric environment, suggesting that the liposomes prepared from milk fat globule membranederived phospholipids were stable and protected the entrapped LF from pepsin hydrolysis. In simulated intestinal fluid, the entrapped LF was more susceptible to hydrolysis by the protease in pancreatin, as shown by changes in the diameter and membrane structure of the liposomes. The release of free fatty acids from the liposomes during digestion in simulated intestinal fluid revealed that the phospholipids in the liposomes were partly hydrolyzed by pancreatic lipase. It was suggested that liposomes may prevent the gastric degradation of $\mathrm{LF}$ and reduce the rate of hydrolysis of LF in intestinal conditions.
\end{abstract}

Key words: lactoferrin, liposome, milk fat globule membrane-derived phospholipid, digestion stability

\section{INTRODUCTION}

Lactoferrin ( $\mathbf{L F})$, a glycoprotein, is present in mammalian milk at an average concentration of $1.4 \mathrm{mg} / \mathrm{mL}$ and exhibits a net positive charge at physiological $\mathrm{pH}$ (Levay and Viljoen, 1995). It is able to bind ferric ions and has bacteriostatic, bactericidal, and fungistatic activities (Palmano et al., 2011). Because LF binds ferric ions with high affinity, it has been proposed as

Received August 21, 2012.

Accepted December 9, 2012.

${ }^{1}$ Corresponding authors: a.m.ye@massey.ac.nz and chengmeiliu@ yahoo.com.cn an antioxidant and has been used in infant formulas (Satué-Gracia et al., 2000). However, it is generally recognized that LF is easily hydrolyzed by the enzymes in the gastrointestinal (GI) tract, which may affect its biological activity (Kuwata et al., 2001). Therefore, the encapsulation of LF into a delivery system, such as an emulsion or liposomes, could be useful in preventing the degradation of LF and maintaining its structural integrity.

A liposome, in which a lipid bilayer encapsulates a fraction of the surrounding aqueous medium, is a selfassembling and cell-resembling colloidal delivery system (Lasic and Papahadjopoulos, 1995). Because of their ability to protect and control the release of bioactive components, the applications of liposomes have focused mainly on the pharmaceutical industry, the cosmetic industry, food science, and agriculture (Taylor et al., 2005; Min et al., 2011; Gabizon et al., 2012). Some reviews have specifically considered the utilization of liposomes in encapsulating bioactive proteins such as LF, which are susceptible to digestion and exhibit low permeability through the intestinal membrane. Trif et al. (2001) demonstrated that the entrapment of human LF in liposomes could prolong its retention at sites of local inflammation, such as the rheumatoid joint. Ishikado et al. (2005) encapsulated LF in multilamellar liposomes and found that liposomal LF might act more effectively than conventional LF in the immune system of rats. Roseanu et al. (2010) also stated that a liposomal formulation of LF could increase its uptake and accumulation into cells, and could probably protect it from degradation. To date, however, most studies have focused on whether the bioactivity of LF is improved by liposomal encapsulation. Little information is available to show whether or not liposomes and the entrapped ingredients maintain their structure during GI digestion (Hermida et al., 2009).

Traditionally, liposomes have been produced from high-cost phospholipids extracted from soybean oil or egg yolk, which may limit their applications. Milk fat globule membrane (MFGM)-derived phospholipids, which are isolated commercially from waste dairy streams such as buttermilk, can be used for liposome 
preparation. They contain approximately $25 \%$ sphingomyelin, and the FA in the chains attached to the phospholipid headgroups are primarily saturated and monounsaturated (Spitsberg, 2005; Singh, 2006). This unique composition of MFGM-derived phospholipids, which is very different from that of the commonly used phospholipids, can play a role in the transport and delivery of active agents in the GI tract (Corredig and Dalgleish, 1998; Roesch et al., 2004). When MFGMderived phospholipids were used in our laboratory, the liposomes prepared had a higher phase-transition temperature, thicker membrane, and lower membrane permeability compared with liposomes prepared from soybean-derived phospholipids (Thompson et al., 2006b). The liposomes prepared from MFGM-derived phospholipids also had improved stability under a range of $\mathrm{pH}$ conditions, at a variety of storage and processing temperatures (Thompson et al., 2006a; Liu et al., 2012). Waninge et al. (2003) also reported that liposome-like vesicles could be produced from simulated MFGM-derived phospholipids. However, the stability to digestion and the structural integrity of liposomes prepared from MFGM-derived phospholipids and of their entrapped contents under GI conditions have not been explored.

In this study, we aimed to extend our previous studies (Thompson et al., 2006a; Liu et al., 2012) and to provide detailed information on the stability of an LF-loaded liposomal system under simulated GI tract conditions. Milk fat globule membrane-derived phospholipids were used to prepare multilayer liposomes by thin-layer dispersion methods, and their properties, including $z$-average diameter, surface charge, entrapment efficiency (EE), and morphology, were evaluated. Furthermore, to assess the stability of the LF-loaded liposomes, lipid hydrolysis and protein release during in vitro digestion were also investigated.

\section{MATERIALS AND METHODS}

\section{Materials}

A phospholipid-rich fraction derived from MFGM (Phospholac 600) was a gift from the Fonterra Cooperative Group Ltd. (Auckland, New Zealand). It contained $\geq 23 \%$ phosphatidylcholine, $\geq 73 \%$ polar lipids, and $\geq 37 \%$ (wt/wt) SFA. Lactoferrin $(\geq 90 \%)$ was purchased from Tatua Co-operative Dairy Company Ltd. (Morrinsville, New Zealand). Pepsin from porcine gastric mucosa (P7000; enzymatic activity of 800 to 2,500 $\mathrm{U} / \mathrm{mg}$ of protein), pancreatin from porcine pancreas [P1750; $4 \times$ US Pharmacopeia (USP) specifications], and bile extract porcine (B8631) were obtained from Sigma-Aldrich (St. Louis, MO). All other chemicals used were of analytical grade and were obtained from Sigma-Aldrich.

\section{Preparation of Liposomes}

A thin-layer dispersion method was used to prepare suspensions of liposomes. Briefly, MFGM-derived phospholipid, cholesterol, Tween-80, and vitamin E were mixed in a mass ratio of 6:1:1.8:0.12, as described in our previous study (Liu et al., 2011). The mixture was well dissolved in absolute ethanol and then evaporated to a thin film under vacuum in a rotary evaporator (R-215; Büchi Labortechnik AG, Flawil, Switzerland) at $60^{\circ} \mathrm{C}$. The dried lipid film was rehydrated with PBS (pH 7.4, $0.05 M)$ containing $\mathrm{LF}$ at $2 \mathrm{mg} / \mathrm{mL}$. Liposomes, with lipid (phospholipid and cholesterol) concentrations of $8 \mathrm{mg} / \mathrm{mL}$ and loaded with the LF suspension, were obtained after 10 min of sonication.

\section{Characterization of LF-Loaded Liposomes}

Determination of $z$-Average Diameter and $\zeta$-Potential. A Malvern Zetasizer Nano ZS instrument (Malvern Instruments Ltd., Malvern, Worcestershire, UK) was used to determine the $z$-average diameter of the LF-loaded liposomes at $20^{\circ} \mathrm{C}$. The relative refractive index [i.e., the ratio of the refractive index of the phospholipid (1.490) to that of the dispersion medium (1.330)] was 1.120. The absorption of the phospholipid was 0.001. Samples were diluted 10 fold in PBS. Mean particle diameters were calculated as the average of at least triplicate measurements. The $\zeta$-potential values of the liposomes were measured using the same instrument and samples were diluted 10 fold with PBS before analysis. An individual $\zeta$-potential measurement was calculated from the mean and the standard deviation of at least 10 readings from each sample.

Determination of $\boldsymbol{E} \boldsymbol{E}$. The percentage of entrapped LF was measured after the free protein had been separated from the liposomes using an ultracentrifuge (Sorvall WX Ultra 100; Thermo Scientific, Asheville, $\mathrm{NC})$ at $396,420 \times g$ for $1 \mathrm{~h}$ at $4^{\circ} \mathrm{C}$. The supernatant was withdrawn and the remaining liposome pellet was washed 3 times with Milli-Q water (water purified by treatment with a Milli-Q apparatus; Millipore Corp., Bedford, MA) followed by resuspension in the same initial volume of PBS. The suspension was then used to measure the amount of LF encapsulated in liposomes by the Kjeldahl method (2100 distilling unit and 2006 digestor block; Tecator AB, Höganäs, Sweden). The total amount of LF in the liposome dispersion before centrifugation was determined using the same method. The EE was calculated using the following equation: 


$$
\mathrm{EE} \%=\frac{\mathrm{W}_{\text {in }}}{\mathrm{W}_{\text {total }}} \times 100,
$$

where $\mathrm{W}_{\text {in }}$ is the analyzed weight of LF encapsulated in the liposomes and $\mathrm{W}_{\text {total }}$ represents the total weight of protein.

Morphology. The morphology of the LF-loaded liposomes prepared from MFGM-derived phospholipids was observed using confocal laser scanning microscopy (CLSM) and thin-section transmission electron microscopy (TEM).

For CLSM, a $500-\mu \mathrm{L}$ sample was stained with $40 \mu \mathrm{L}$ of Nile red solution $(0.25 \mathrm{mg} / \mathrm{mL})$ and $80 \mu \mathrm{L}$ of fast green solution $(1 \mathrm{mg} / \mathrm{mL})$, which were used to stain the phospholipids and the protein, respectively. The mixed solution was placed on a concave confocal microscope slide and $50 \mu \mathrm{L}$ of agarose $(0.5 \%)$ was added to fix the sample. The sample was then covered with a coverslip and dried at room temperature in the dark. Images of the sample were acquired using a confocal laser scanning microscope (Leica DM6000 B; Leica Microsystems Heidelberg GmbH, Heidelberg, Germany) with a $63 \times$ magnification lens.

Thin-section TEM was used to provide visual confirmation of the microstructure of the liposomes. Lactoferrin-loaded liposomes were centrifuged at 396,420 $\times g$ for $1 \mathrm{~h}$ at $4^{\circ} \mathrm{C}$ using an ultracentrifuge (Sorvall WX Ultra 100; Thermo Scientific) and the pellet at the bottom was collected. The pellet was then injected into a 3\% low-temperature-gelling agarose tube, which was sealed with the same agarose, which had been freshly prepared. The sample-containing tube was fixed in $3 \%$ glutaraldehyde in $0.2 \mathrm{M}$ sodium cacodylate buffer for $24 \mathrm{~h}$ at room temperature. The sample was washed 3 times with $0.2 \mathrm{M}$ sodium cacodylate buffer for $2 \mathrm{~h}$ and was then left in $1 \%$ osmium tetroxide overnight at room temperature. The 3 buffer washes were repeated. The sample was then dehydrated using an acetone/water series $(25,50,75,95$, and 100\%), keeping it in each gradient for $30 \mathrm{~min}$ and in the $100 \%$ acetone for $2 \mathrm{~h}$. It was first embedded with an acetone:resin (Procure 812; ProSciTech Pty Ltd., Thuringowa, Australia) mixture (50:50) on a stirrer overnight and was then moved to $100 \%$ resin for $32 \mathrm{~h}$ at room temperature. The resin was replaced with fresh $100 \%$ resin every 8 or $12 \mathrm{~h}$. The sample was finally mounted in $100 \%$ fresh resin at $60^{\circ} \mathrm{C}$ for $48 \mathrm{~h}$.

Sections $1 \mu \mathrm{m}$ in thickness were cut from trimmed resin blocks using a glass knife and an ultramicrotome (Leica Mikrosysteme Handelsges.m.b.H., Vienna, Austria). They were heat mounted onto a glass slide, stained with $0.05 \%$ toluidine blue, and viewed under a light microscope (Olympus BX51; Olympus Corp.,
Tokyo, Japan). Areas of interest were chosen for examination using TEM (CM10; Philips, Eindhoven, the Netherlands).

Ultra-thin sections (100 nm) were cut using a diamond knife and an ultramicrotome, followed by collection on a copper grid. The sections were stained with saturated uranyl acetate in 50\% ethanol for 4 min and then by lead citrate for another $4 \mathrm{~min}$. The specimens were examined using TEM.

Polyacrylamide Gel Electrophoresis. Sodium dodecyl sulfate PAGE was used to confirm the entrapped LF and the total LF in the liposome suspension. Lactoferrin-loaded liposomes were centrifuged at 396,420 $\times g$ for $1 \mathrm{~h}$ at $4^{\circ} \mathrm{C}$ using an ultracentrifuge (Sorvall WX Ultra 100; Thermo Scientific). The supernatants were carefully sucked out by syringe and the pellet was resuspended in the same volume of PBS. Then, the supernatants, the pellet solution, the liposome suspension before centrifugation, and LF solution (LF dissolved in PBS, $2 \mathrm{mg} / \mathrm{mL}$ ) were analyzed using SDS-PAGE.

Briefly, the pellet solution and the LF-loaded liposomes were added to Triton X-100 solution $[10 \%$ in PBS, sample:Triton X-100 = 1:0.1 ( $\mathrm{vol} / \mathrm{vol})]$, whereas the supernatants and the LF solution were mixed well with PBS in a volume ratio of 1:0.1. These samples were then treated with SDS buffer $(0.5 M$ Tris, $2.0 \%$ SDS, 0.04\% Coomassie Brilliant Blue G-250, and 0.05\% $\beta$-mercaptoethanol, $\mathrm{pH} 6.8$ ) in a volume ratio of $1: 1.5$ and were reduced by heating in a boiling water bath $\left(\sim 95^{\circ} \mathrm{C}\right)$ for $5 \mathrm{~min}$. After cooling to room temperature, $10-\mu \mathrm{L}$ samples were loaded on to a precast gel (10 to 20\% Tris-tricine/peptide; Bio-Rad Laboratories Inc., Richmond, CA) and were run in a Mini-Protean system (Bio-Rad Laboratories Inc.) at $200 \mathrm{~V}$ using a Bio-Rad power supply unit (model 1000/500; Bio-Rad Laboratories Inc.). The gel was then stained with staining solution (0.03\% Coomassie Blue R-250, $10 \%$ glacial acetic acid, and $20 \%$ isopropanol) for about $40 \mathrm{~min}$ and destained in destaining solution $(10 \%$ glacial acetic acid and $10 \%$ isopropanol) until the background had faded, which was then followed by scanning on a molecular imager (Universal Hood II; Bio-Rad Laboratories Inc.).

\section{Preparation of Simulated Gastric and Intestinal Fluids}

Simulated gastric fluid (SGF) and simulated intestinal fluid (SIF) were prepared as described in Singh and Sarkar (2011). Sodium chloride (2 g) was dissolved in Milli-Q water, which was followed by the addition of 7 $\mathrm{mL}$ of concentrated hydrochloric acid, and the SGF was obtained by diluting the solution to $1 \mathrm{~L}$ and adjusting the $\mathrm{pH}$ to 1.2. The SIF contained $6.8 \mathrm{~g}$ of dipotassium 
phosphate and $190 \mathrm{~mL}$ of $0.1 M$ sodium hydroxide, with $150 \mathrm{~m} M$ sodium chloride and $30 \mathrm{~m} M$ calcium chloride; this mixture was diluted to $1 \mathrm{~L}$ and the $\mathrm{pH}$ was adjusted to 7.4 ; the bile salt concentration was $0.2 \mathrm{mg} / \mathrm{mL}$. Before in vitro liposome digestion, the SGF and the SIF were incubated at $37^{\circ} \mathrm{C}$ with continuous agitation at 95 rpm in a temperature-controlled water bath (Lab-Line shaker bath, model LZ33070; Barnstead International, Dubuque, IA). The $\mathrm{pH}$ and the temperature were continuously monitored and controlled.

\section{Stability of LF-Loaded Liposomes During Digestion}

Digestion of the LF-loaded liposomes was carried out in SGF and SIF separately according to the following procedures. Liposomes were incubated in SGF containing pepsin $(0.032 \mathrm{mg} / \mathrm{mL})$ or in SIF containing pancreatin $(0.032 \mathrm{mg} / \mathrm{mL})$ at a volume ratio of $1: 3$. The mixture $(60 \mathrm{~mL})$ containing SGF had a $\mathrm{pH}$ of 1.5 and that containing SIF had a $\mathrm{pH}$ of 7.4. These mixtures were incubated in a shaking water bath (95 rpm) at $37^{\circ} \mathrm{C}$ and subsamples $(4 \mathrm{~mL})$ were taken for analysis at different time intervals $(0,1,5,15,30,60$, and 120 $\min )$.

Trends in the $z$-average diameter, the $\zeta$-potential, and the structural alteration of the liposomes during digestion were monitored using a Malvern Zetasizer Nano ZS instrument and thin-section TEM, as described above.

\section{Lipolysis}

In vitro lipid digestion was monitored as described by Sek et al. (2002) using a pH-stat titration technique (TIM 856; Radiometer Pacific Pty Ltd., Melbourne, Australia). The LF-loaded liposomes $(8 \mathrm{~mL})$ were mixed with $24 \mathrm{~mL}$ of SIF and the $\mathrm{pH}$ was adjusted to 7.4 , before heating to $37^{\circ} \mathrm{C}$. The stability of the liposomal MFGM-derived phospholipids was determined by measuring the FFA released from the samples after the addition of pancreatin $(0.032 \mathrm{mg} / \mathrm{mL})$, using $0.05 M$ sodium hydroxide to reach an endpoint of $\mathrm{pH}$ 7.4. Lipid digestion without enzyme was also carried out using the same procedures. A standard curve was constructed, using the same titration method to measure the FFA present in liposomes and SIF [1:3 (vol/vol)] with known concentrations (0 to $15,000 \mu \mathrm{mol}$ ) of oleic acid.

\section{Protein Hydrolysis}

The time-dependent hydrolysis of protein during digestion was examined using SDS-PAGE, as described by Ye et al. (2011). Samples were withdrawn from the mixed solution into Eppendorf vials containing $0.8 \mathrm{M}$ sodium carbonate [sample:sodium carbonate $=1: 0.25$ ( $\mathrm{vol} / \mathrm{vol}$ )] after digestion in SGF for $0,1,5$, and 60 min, which was followed by the addition of Triton X-100 solution $[10 \%$ in PBS, sample:Triton X-100 = 1:0.1 ( $\mathrm{vol} / \mathrm{vol})]$. For the samples digested in SIF, the enzyme was inactivated by adding $0.05 M$ hydrochloric acid [sample:hydrochloric acid $=1: 0.04(\mathrm{vol} / \mathrm{vol})$ ] and heating in a boiling water bath $\left(\sim 95^{\circ} \mathrm{C}\right)$ for $30 \mathrm{~s}$. As a control, LF solution (LF dissolved in PBS, $2 \mathrm{mg} / \mathrm{mL}$ ) was also digested in SGF or SIF for 0, 1, 5, and $60 \mathrm{~min}$. The same procedures as described above were used for loading the samples and running the gels.

To estimate whether the LF entrapped in the liposomes was affected by the amount of enzyme, different concentrations of pepsin $(0,0.0032,0.032,0.32$, 3.2 , and $10 \mathrm{mg} / \mathrm{mL}$ ) were used in the SGF digestion. Lactoferrin-loaded liposomes were withdrawn after 60 min of digestion and were measured by SDS-PAGE.

\section{Statistical Analysis}

All measurements were replicated at least 3 times. The results were evaluated statistically for significance $(P \leq 0.05)$ using ANOVA (Manconi et al., 2010) and SPSS software version 18.0 (IBM Corp., Chicago, IL). All data were expressed as means \pm standard deviation.

\section{RESULTS}

\section{Physicochemical Properties of LF-Loaded Liposomes}

The $z$-average diameter and the $\zeta$-potential of the LF-loaded liposomes prepared from MFGM-derived phospholipids were $1,369 \pm 109 \mathrm{~nm}$ and $-13.0 \pm 0.4$ $\mathrm{mV}$, respectively, with a polydispersity index (PDI) of $0.28 \pm 0.02$. The $\mathrm{EE}$ of the LF in the liposomes was calculated to be $46.4 \pm 8.7 \%$.

The microstructure of the LF-loaded liposomes was observed by CLSM and thin-section TEM. As shown in the CLSM images (Figure 1), the liposomes were well distributed and were even in size (consistent with the PDI value). Most of the liposomes appeared to be smooth and spherical in shape. In the higher magnification image (Figure 1b), a green color (indicating protein) was observed within some of the liposomal vesicles, indicating entrapped LF, although this was not found for all liposomes (see arrow).

According to the TEM results (Figure 2), the liposomes appeared to be primarily between 1,000 and $1,500 \mathrm{~nm}$ in size, which was in agreement with the average diameter measurements. Although some of the liposomes seemed to be irregular, many were spherical 



Figure 1. Confocal laser scanning microscopy images of lactoferrin-loaded liposomes prepared from milk fat globule membrane-derived phospholipids: (a) low magnification; (b) high magnification. Arrows represent lactoferrin encapsulated in liposomes. Color version available in the online PDF.

or ellipsoidal in shape (Figure 2a) and had considerably integrated structures. A very small proportion of the vesicles were liposome within liposome, often referred to as multivesicular liposomes. In particular, bilamellar vesicles were clearly observed, with their inner membrane being much thicker than their outer membrane (Figure 2b).

The SDS-PAGE analysis of the LF entrapped in the liposomes showed clearly no apparent difference in protein content between the LF solution and the LF in the liposome dispersion (Figure 3, lanes 1 and 2). After centrifugation of the liposome dispersion, the proportion of LF encapsulated in the liposomes (lane 3 vs. lane 2) was about $40 \%$, which was close to the results of the EE measurement using the Kjeldahl method.

\section{Changes in Size of Liposomes During Digestion}

The mean particle diameters of the LF-loaded liposomes (Figure 4) were measured as a function of time during digestion in SGF or in SIF. In the absence of enzyme, the average diameter of the LF-loaded liposomes was about $1,050 \mathrm{~nm}$, with a PDI of approximately 0.5. However, the average diameter decreased to about 500 $\mathrm{nm}$ when the liposomes were mixed with SGF in the absence of pepsin and did not change significantly during digestion for $2 \mathrm{~h}$ in SGF in the presence of pepsin. The average diameter of the LF-loaded liposomes increased markedly in the first $1 \mathrm{~min}$ of digestion in SIF (about 2,389 $\pm 215 \mathrm{~nm}$; PDI $=0.69 \pm 0.11$ ), with a gradual decrease thereafter.
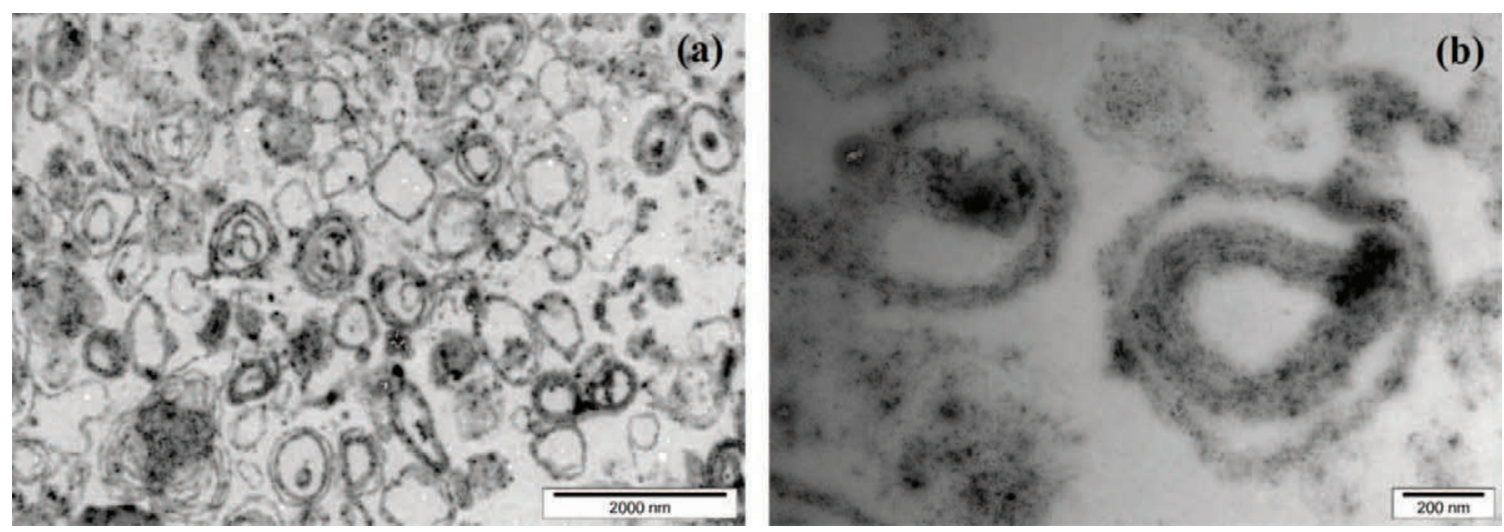

Figure 2. Thin-section transmission electron microscopy images of lactoferrin-loaded liposomes prepared from milk fat globule membranederived phospholipids: (a) low magnification; (b) high magnification. 


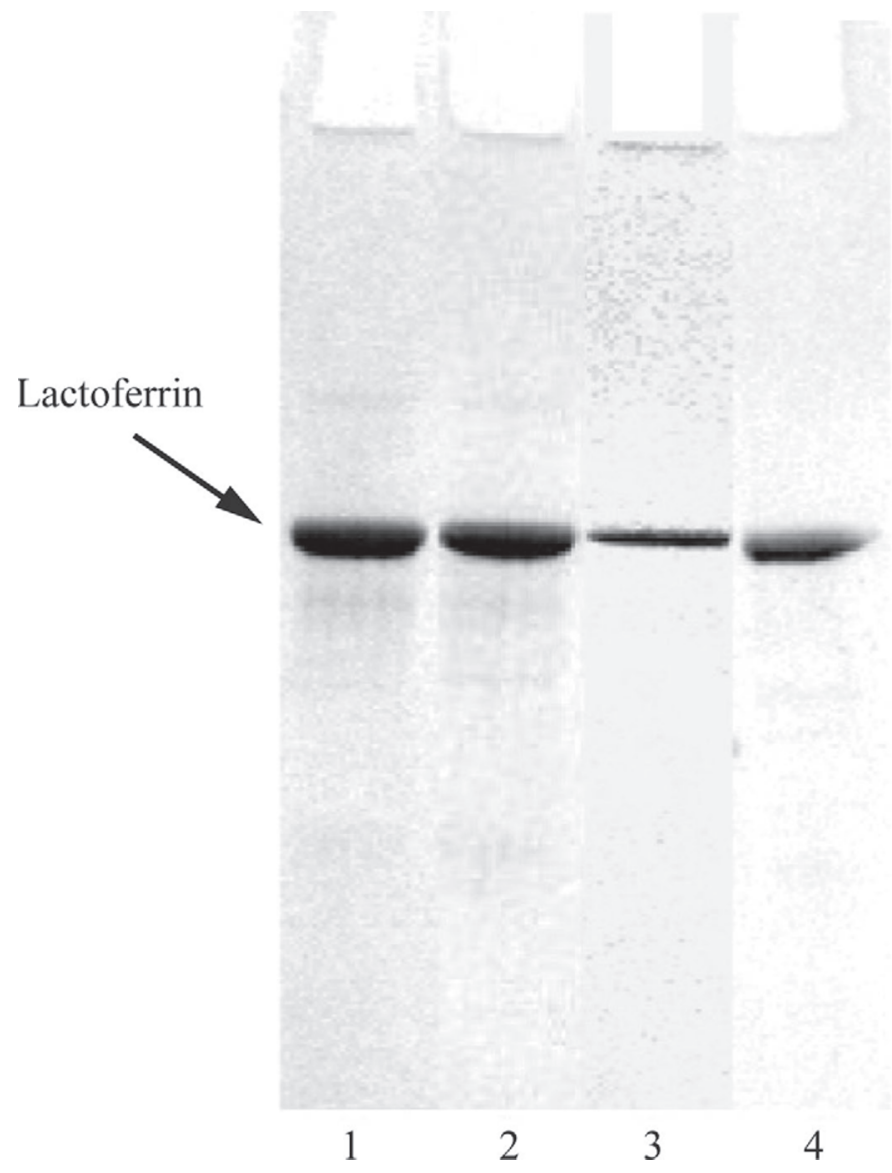

Figure 3. Reducing tricine-SDS-PAGE patterns of lactoferrin solution (lane 1), lactoferrin entrapped in liposomes (lane 2), pellet solution (lane 3, liposomes centrifuged at 396,420 $\times \mathrm{g}$ for $1 \mathrm{~h}$ and pellet obtained resuspended in PBS), and supernatant (lane 4, liposomes centrifuged at $396,420 \times g$ for $1 \mathrm{~h}$ ).

\section{Changes in $\zeta$-Potential of Liposomes During Digestion}

The $\zeta$-potentials were measured to determine the changes in the surface charge of the liposomes during digestion under simulated GI tract conditions (Figure 5). In SGF, the $\zeta$-potential of the LF-loaded liposomes was $+9.2 \pm 1.0 \mathrm{mV}$ in the absence of pepsin and was close to zero in the presence of pepsin. However, in SIF, the $\zeta$-potential of the LF-loaded liposomes was -44.2 $\pm 0.4 \mathrm{mV}$ in the absence of pancreatic lipase and no obvious changes were observed during digestion in SIF containing pancreatic lipase.

\section{Morphology of Liposomes}

Thin-section TEM images of LF-loaded liposomes digested in SGF and in SIF for 120 min are shown in Figure 6. After digestion in SGF (Figures 6a and 6c), the distribution of liposomes was nonuniform (about 100 to $2,000 \mathrm{~nm}$ ) and some small particles were inter-

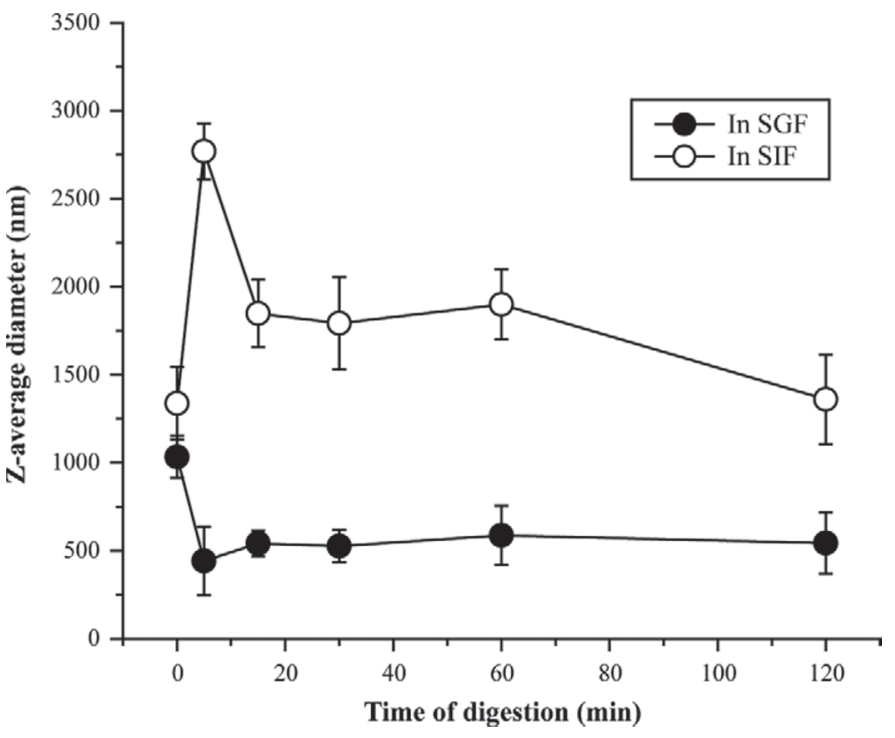

Figure 4. Changes in z-average diameter of lactoferrin-loaded liposomes during digestion in simulated gastric fluid (SGF; $\bullet$ ) and simulated intestinal fluid (SIF; $\bigcirc)$. Each data point is the average \pm SD of 3 determinations on 3 separate samples.

spersed within larger particles. Most of the liposomes exhibited dense structures and rough surfaces, but had integrated structures. However, after digestion in SIF, the liposomes were irregular in shape, there were fewer intact liposomes remaining (Figure 6b), and the liposomal membrane was obviously damaged, with some

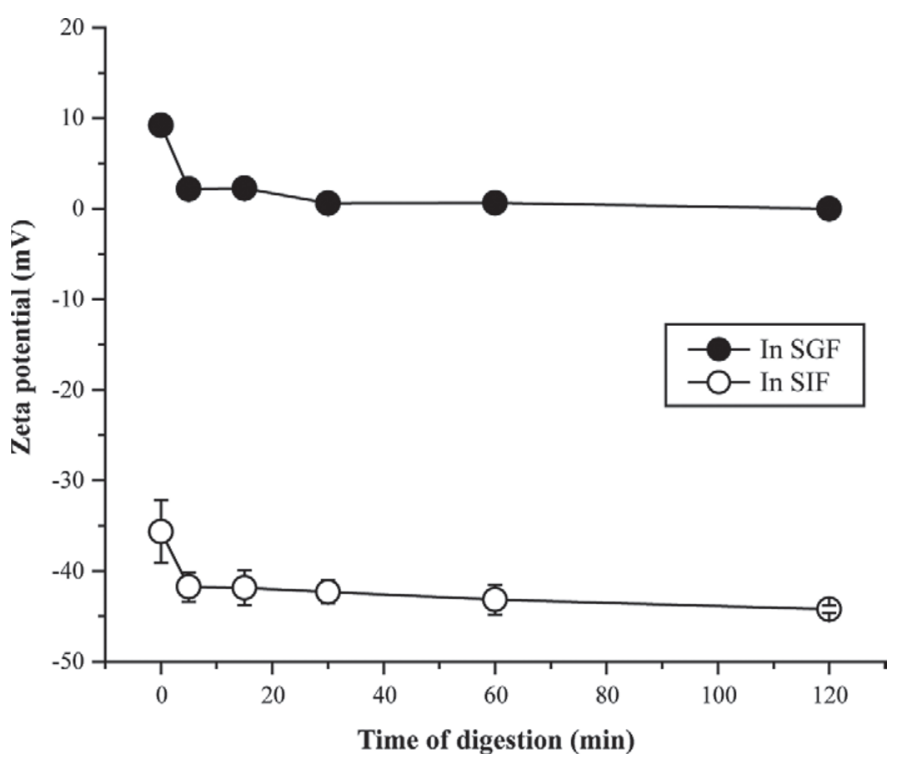

Figure 5. Changes in $\zeta$-potential of lactoferrin-loaded liposomes during digestion in simulated gastric fluid $(\mathrm{SGF} ; \mathbf{0})$ and simulated intestinal fluid (SIF; $\bigcirc$ ). Each data point is the average $\pm \mathrm{SD}$ of 3 determinations on 3 separate samples. 

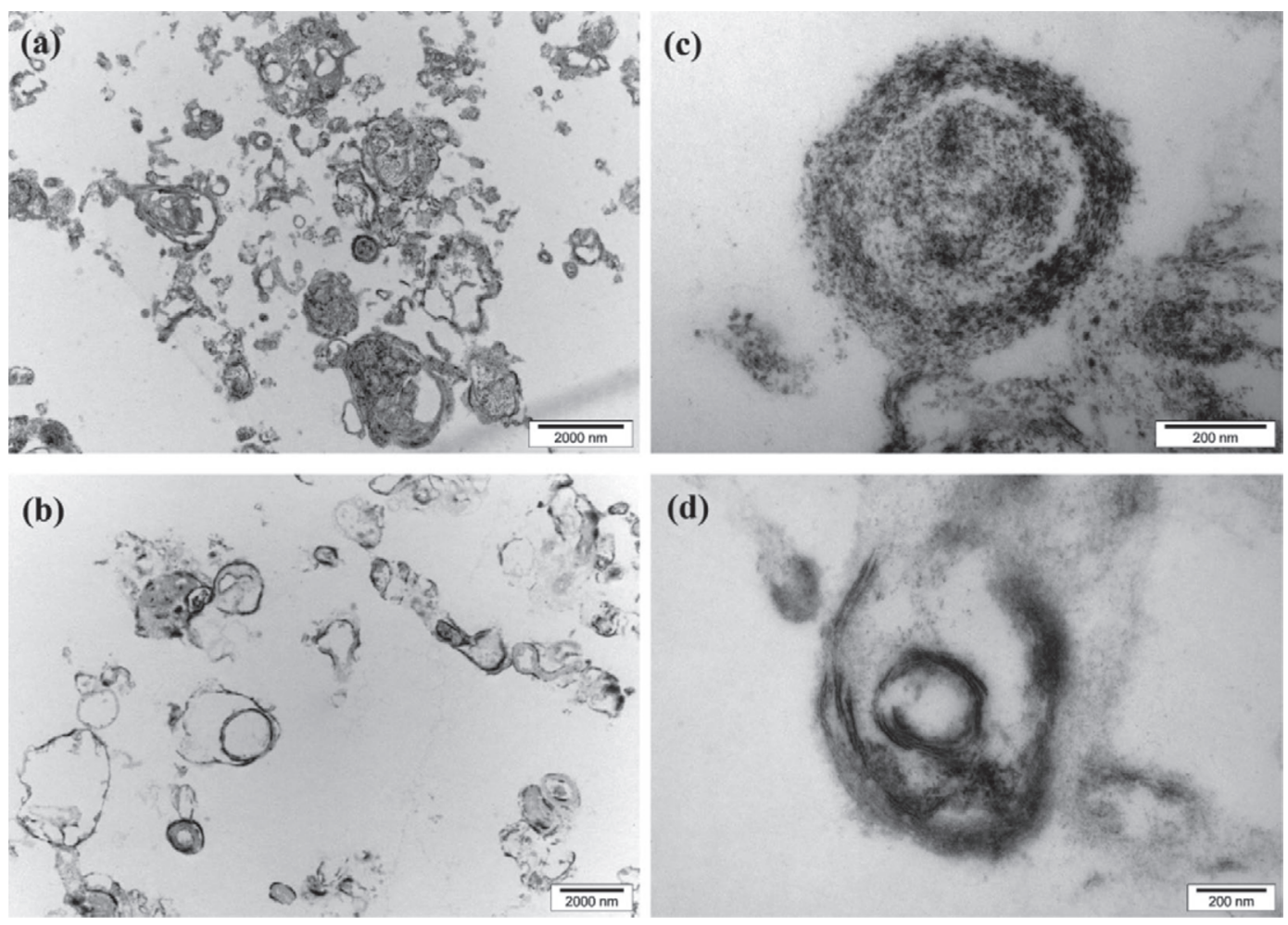

Figure 6. Thin-section transmission electron microscopy images of lactoferrin-loaded liposomes after digestion for 120 min in simulated gastric fluid [(a) low magnification; (c) high magnification] and simulated intestinal fluid [(b) low magnification; (d) high magnification].

of the inner membranes also appearing to be broken (Figure 6d).

\section{Lipolysis of Liposomes}

The lipolysis of the bilayers in the LF-loaded liposomes prepared from MFGM-derived phospholipids under SIF conditions was quantified by determining the release of FFA (Figure 7). Enzymatic lipolysis of the liposomes increased gradually during the digestion procedure $(45.5 \pm 4.3 \mu M)$, whereas only slight changes were observed in the FFA released from liposomes digested in the absence of enzyme.

\section{Stability of Encapsulated Protein}

The SDS-PAGE was used to evaluate the stability of the LF-loaded liposomes during digestion in SGF and in SIF. This information could also indicate the stability of the liposomal membrane against enzymatic hydrolysis and the integrity of the liposomes in the GI tract. Figure 8a shows the intensity of the LF bands as a function of digestion time in SGF and SIF. The LF solution was completely hydrolyzed by pepsin after 1 min of digestion in SGF. However, about $40 \%$ of the $\mathrm{LF}$ band remained in the liposome sample after $1 \mathrm{~min}$ of digestion [lane 1 (control) and lane 2]. This amount
(40\%) of LF was similar to the amount of LF entrapped in the liposomes, as shown in Figures 1 and 3. Furthermore, the amount of LF did not decrease during further digestion up to 60 min (lane 3 and lane 4),

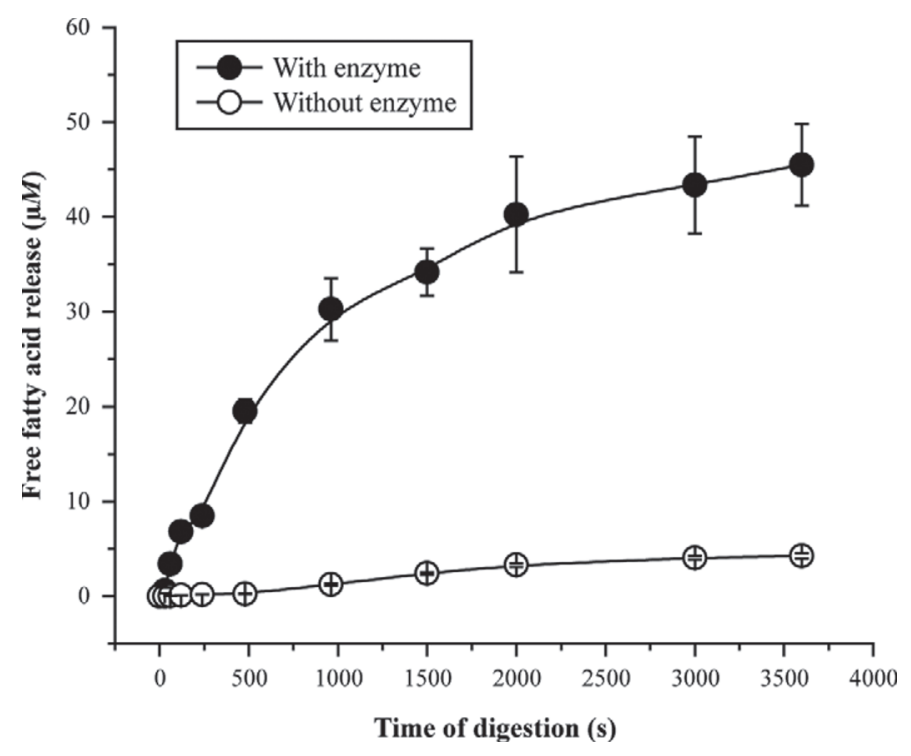

Figure 7. Free FA released from lactoferrin-loaded liposomes as a function of time during digestion in simulated intestinal fluid: $(\bullet)$ with pancreatin; $(\bigcirc)$ without pancreatin. Each data point is the average \pm $\mathrm{SD}$ of the determination on 3 separate samples. 

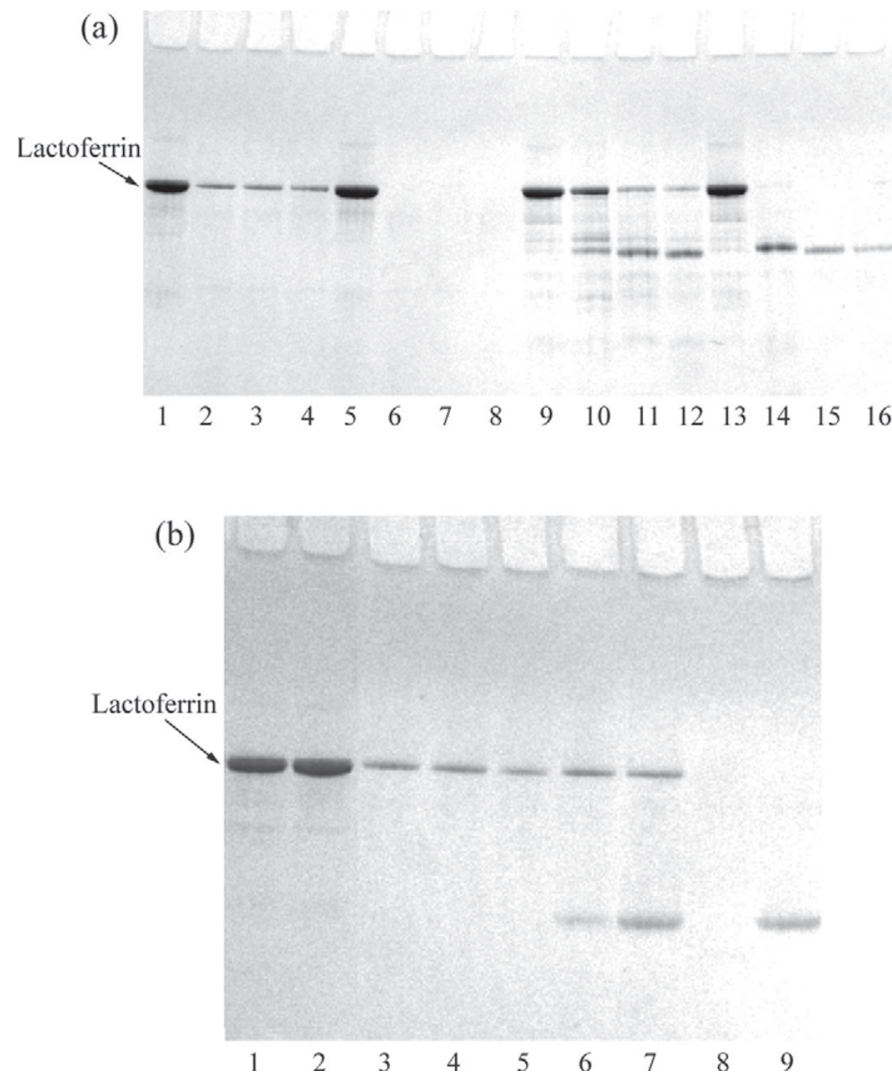

Figure 8. Reducing tricine-SDS-PAGE patterns. (a) Lactoferrin (LF)-loaded liposomes and LF solution digested in simulated gastric fluid (SGF) and in simulated intestinal fluid (SIF), respectively: lanes 1 to 4 = liposomes digested in SGF for $0,1,5$, and $60 \mathrm{~min}$, respectively; lanes 5 to $8=\mathrm{LF}$ solution digested in SGF for $0,1,5$, and 60 min, respectively; lanes 9 to $12=$ liposomes digested in SIF for $0,1,5$, and $60 \mathrm{~min}$, respectively; lanes 13 to $16=\mathrm{LF}$ solution digested in SIF for $0,1,5$, and $60 \mathrm{~min}$, respectively. (b) Lactoferrin-loaded liposomes and LF solution digested in SGF containing different concentrations of pepsin: lanes 1, 8, and $9=\mathrm{LF}$ solution digested in SGF with pepsin concentrations of $0,0.0032$, and $10 \mathrm{mg} / \mathrm{mL}$, respectively; lanes 2 to 7 $=\mathrm{LF}$-loaded liposomes digested in SGF with pepsin concentrations of $0,0.0032,0.032,0.32,3.2$, and $10 \mathrm{mg} / \mathrm{mL}$ pepsin, respectively.

which indicated that the LF entrapped in the liposomal membrane was not hydrolyzed by pepsin under SGF conditions.

In SIF, the LF band in the liposome sample gradually decreased with an increase in the digestion time and only a small/fuzzy band could be observed after 60 min of digestion (lane 12). In contrast, for LF solution, the LF band had completely disappeared after $1 \mathrm{~min}$ of digestion in SIF (lane 14). Bands with lower molecular weight were observed after digestion, indicating that the LF in both the liposome and the solution had been hydrolyzed in SIF, but that the LF in the liposome was less susceptible than the LF in solution.

Figure $8 \mathrm{~b}$ shows the stability of LF entrapped in liposomes when digested in SGF for 60 min with different pepsin concentrations (from 0 to $10 \mathrm{mg} / \mathrm{mL}$ ). For all the liposomal samples after digestion, no difference in the intensities of the LF bands was observed, even though the pepsin concentration was increased sharply from 0.0032 to $10 \mathrm{mg} / \mathrm{mL}$ (band 3 to band 7). However, for the LF solution samples, no intact LF was observed after digestion for 60 min with both a low concentration $(0.0032 \mathrm{mg} / \mathrm{mL})$ and a high concentration $(10 \mathrm{mg} /$ $\mathrm{mL}$ ) of pepsin (lanes 8 and 9).

\section{DISCUSSION}

After mixing with SGF, the diameter of all the digested liposome samples was smaller than that of the original liposomes (Figure 4). Nacka et al. (2001) obtained a similar result and stated that the liposomal diameter decreased gradually to reach about $50 \%$ of the initial diameter after storage at $\mathrm{pH} 1.5$ for $30 \mathrm{~min}$. In SGF, membrane shrinkage probably causes the decrease in the size of the liposomes, because of water leaking from the inside of the liposomes $(\mathrm{pH} 7.4$, low osmolarity) to the outside ( $\mathrm{pH} 1.5$, high osmolarity). However, the slight change in the diameter during SGF digestion suggested that the liposomes could retain their integrity and that pepsin did not further hydrolyze and damage the phospholipid membrane of the liposomes.

The TEM results (Figure 6) confirmed that, although the acidic conditions caused an alteration in shape, most of the bilayers retained their structures intact. This may be attributed to the well-organized assembly of the phospholipids in the liposomes and to the addition of cholesterol, which was effective in protecting the liposomes from membrane damage and pepsin digestion under low pH conditions (Hwang et al., 2010).

In addition, the lamellae of the LF-loaded liposomes were thick, which may be attributed to the electrostatic interaction between the positively charged LF and the negatively charged groups of the phospholipids in the liposomal membrane (Kimelberg et al., 1970). The positively charged protein would be localized on the surface polar headgroups of the phospholipid molecules, rather than in the hydrophobic interior of the bilayers, resulting in an increase in the width of the liposomal membrane and rough spherical surfaces. Furthermore, the EE of LF was about $40 \%$. Trif et al. (2007), who used a freeze-thaw method to prepare LFloaded liposomes, also reported an EE of approximately $40 \%$. The entrapped LF appeared to be protected from pepsin hydrolysis during digestion in SGF, independent of the concentration of enzyme (Figure 8). This suggested that the integrity of the bilayer membrane of the liposome was maintained and that this bilayer structure prevented pepsin from permeating into the core of the liposome even at high pepsin concentration (Figure $8 \mathrm{~b}$ ). Our result was in agreement with results 
of Bardonnet et al. (2009) and Smith et al. (2010), who found that liposomes remained relatively stable when exposed to simulated gastric conditions and that $80 \%$ of the encapsulated alkaline phosphatase retained its original activity.

The effects of SIF, which contained bile salts and pancreatin, on the liposomes were more severe. The average diameters of the LF-loaded liposomes were notably increased in the initial stages of digestion, followed by a gradual decrease in the later stages (Figure $4)$, the $\zeta$-potential became more negative during the digestion period (Figure 5), and fewer liposomes with intact membranes remained (Figure 6). These changes may be attributed to the hydrolysis of the phospholipids in the liposomes by pancreatin, which contains lipase, phospholipase $\mathrm{A}_{2}$, and cholesterol esterase. Pancreatic lipase has been found to catalyze the hydrolysis of 1-linked FA of phospholipids, releasing FA and 1-acyl lysophospholipids (de Haas et al., 1965). The release of FFA (Figure 7) from the liposomes digested under SIF conditions confirmed the lipolysis of the liposomal membrane. In addition, the phospholipase $\mathrm{A}_{2}$ in pancreatic extracts could catalyze the $s n-2$ ester bond hydrolysis of phospholipids to glycerophosphoric acids and 2-acyl lysophospholipids, which are well known for their ability to destabilize phospholipid bilayers. Furthermore, cholesterol esterase, also known as bile salt-stimulated lipase, is capable of hydrolyzing phospholipids (Howles et al., 1996). Therefore, the organized structure of the liposomal bilayers could be modified by these 3 enzymes, resulting in aggregation of the liposomes, as indicated by the increase in the particle diameter.

The decrease in the average diameter of the liposomes during digestion in SIF may have been because the detergent-like property of the bile salts disrupted the structure of the phospholipid membrane (O'Connor et al., 1985). Kokkona et al. (2000) also found that the mean diameter of dipalmitoylphosphatidylcholinebased liposomes decreased by $50 \%$ after digestion for 24 $\mathrm{h}$ in the presence of bile salts. In addition, the formation of micelles by FA, bile salts, and phospholipids after a long digestion time in SIF could also decrease the average diameter.

The hydrolysis of MFGM-derived phospholipids by pancreatic lipase also affects the surface charge of the vesicle. Lysophospholipids, which are one of the hydrolysis products of phospholipids, exhibit stronger polarity than their parent phospholipids in the liposomes. These hydrolysis products may have been retained at the surface of the liposomes or formed vesicles with bile salts after disruption of the liposomes, resulting in a greater negative surface charge after digestion in SIF.

Phospholipids are specifically degraded by pancreatic lipase and phospholipase $\mathrm{A}_{2}$, with a concomitant release of FA and acyl lysophospholipids (Nishizuka, 1992). However, according to Hunt and Jones (1984), a significant synergism in the combined effects of bile salts and phospholipase could also disrupt the phospholipids of the liposomal membrane and release FA.

Because of the hydrolysis of phospholipids and protein by lipase and the protease in pancreatin, respectively, it was reasonable to expect a gradual degradation of the LF released from the damaged liposomes. However, some LF still remained in the liposome suspension after 60 min of digestion in SIF (Figure 8), whereas no intact $\mathrm{LF}$ remained in the LF solution after digestion for the same period of time. To some extent, this indicated that some entrapped LF in liposomes could survive under SIF digestion.

According to Troost et al. (2001), about 60 to $80 \%$ of the $20 \%$ iron-saturated LF and $100 \%$ iron-saturated LF remained intact after passage through the stomach of healthy adults. However, they also stated that dietary recombinant human LF was digested in the upper GI tract and did not reach the colon (Troost et al., 2002). Brock (2012) also reported that the maturated digestive system resulted in complete digestion of lactoferrin in the upper GI tract of human adults. In our present study, LF entrapped in liposomes was protected and retarded degradation in SGF and SIF conditions, respectively.

\section{CONCLUSIONS}

The present results provide useful information on the stability of LF-loaded liposomes prepared from MFGMderived phospholipids under SGF and SIF conditions. No change occurred in the entrapped LF when the liposome samples were digested in a simulated gastric environment as a function of time and pepsin concentration. The liposomes prepared from MFGM-derived phospholipids were stable and protected the entrapped LF from pepsin hydrolysis. Under simulated intestinal conditions, the LF entrapped in the liposomes was more susceptible to hydrolysis by the protease in pancreatin, as shown by changes in the diameter and the membrane structure of the liposomes. This indicated that encapsulation in the liposomes may prevent gastric degradation of LF and reduce the rate of hydrolysis of LF in intestinal conditions.

\section{ACKNOWLEDGMENTS}

We gratefully thank the Manawatu Microscopy and Imaging Centre at Massey University (Palmerston North, New Zealand) for providing the CLSM and TEM images. W. Liu wishes to thank Riddet Institute (Palmerston North, New Zealand) for help during her study. This research was supported by the National 
Natural Science Foundation of China (Beijing, China; 21266021), the Project of State Key Laboratory of Food Science and Technology, Nanchang University (Jiangxi, P.R. China; SKLF-TS-201115), and Jiangxi Province Postgraduate Innovation Fund (YC2011-B005).

\section{REFERENCES}

Bardonnet, P.-L., V. Faivre, P. Boullanger, M. Ollivon, and F. Falson. 2009. Glycosylated liposomes against Helicobacter pylori: Behavior in acidic conditions. Biochem. Biophys. Res. Commun. 383:48-53.

Brock, J. H. 2012. Lactoferrin-50 years on. Biochem. Cell Biol. $90: 245-251$.

Corredig, M., and D. G. Dalgleish. 1998. Characterization of the interface of an oil-in-water emulsion stabilized by milk fat globule membrane material. J. Dairy Res. 65:465-477.

de Haas, G. H., L. Sarda, and J. Roger. 1965. Positional specific hydrolysis of phospholipids by pancreatic lipase. Biochim. Biophys. Acta 106:638-640.

Gabizon, A., H. Shmeeda, and T. Grenader. 2012. Pharmacological basis of pegylated liposomal doxorubicin: Impact on cancer therapy. Eur. J. Pharm. Sci. 45:388-398.

Hermida, L. G., M. Sabés-Xamaní, and R. Barnadas-Rodríguez. 2009. Combined strategies for liposome characterization during in vitro digestion. J. Liposome Res. 19:207-219.

Howles, P. N., C. P. Carter, and D. Y. Hui. 1996. Dietary free and esterified cholesterol absorption in cholesterol esterase (bile salt-stimulated lipase) gene-targeted mice. J. Biol. Chem. 271:7196-7202.

Hunt, G. R., and I. C. Jones. 1984. Application of ${ }^{1} \mathrm{H}-\mathrm{NMR}$ to the design of liposomes for oral use. Synergistic activity of bile salts and pancreatic phospholipase $\mathrm{A}_{2}$ in the induced permeability of small unilamellar phospholipid vesicles. J. Microencapsul. 1:113-122.

Hwang, J.-S., Y.-L. Tsai, and K.-C. Hsu. 2010. The feasibility of antihypertensive oligopeptides encapsulated in liposomes prepared with phytosterols- $\beta$-sitosterol or stigmasterol. Food Res. Int. 43:133-139.

Ishikado, A., H. Imanaka, T. Takeuchi, E. Harada, and T. Makino. 2005. Liposomalization of lactoferrin enhanced its anti-inflammatory effects via oral administration. Biol. Pharm. Bull. 28:17171721.

Kimelberg, H. K., C. P. Lee, A. Claude, and E. Mrena. 1970. Interactions of cytochrome $c$ with phospholipid membranes. I. Binding of cytochrome $c$ to phospholipid liquid crystals. J. Membr. Biol. $2: 235-251$

Kokkona, M., P. Kallinteri, D. Fatouros, and S. G. Antimisiaris. 2000. Stability of SUV liposomes in the presence of cholate salts and pancreatic lipases: Effect of lipid composition. Eur. J. Pharm. Sci. 9:245-252.

Kuwata, H., K. Yamauchi, S. Teraguchi, Y. Ushida, Y. Shimokawa, T. Toida, and H. Hayasawa. 2001. Functional fragments of ingested lactoferrin are resistant to proteolytic degradation in the gastrointestinal tract of adult rats. J. Nutr. 131:2121-2127.

Lasic, D. D., and D. Papahadjopoulos. 1995. Liposomes revisited. Science 267:1275-1276.

Levay, P. F., and M. Viljoen. 1995. Lactoferrin: A general review. Haematologica 80:252-267.

Liu, W., W.-L. Liu, C.-M. Liu, J.-H. Liu, S.-B. Yang, H.-J. Zheng, H.W. Lei, R. Ruan, T. Li, Z.-C. Tu, and X.-Y. Song. 2011. Mediumchain fatty acid nanoliposomes for easy energy supply. Nutrition 27:700-706.

Liu, W., A. Ye, C. Liu, W. Liu, and H. Singh. 2012. Structure and integrity of liposomes prepared from milk- or soybean-derived phospholipids during in vitro digestion. Food Res. Int. 48:499-506.

Manconi, M., S. Mura, M. L. Manca, A. M. Fadda, M. Dolz, M. J. Hernandez, A. Casanovas, and O. Díez-Sales. 2010. Chitosomes as drug delivery systems for C-phycocyanin: Preparation and characterization. Int. J. Pharm. 392:92-100.
Min, B., J. C. Cordray, and D. U. Ahn. 2011. Antioxidant effect of fractions from chicken breast and beef loin homogenates in phospholipid liposome systems. Food Chem. 128:299-307.

Nacka, F., M. Cansell, J. P. Gouygou, C. Gerbeaud, P. Méléard, and B. Entressangles. 2001. Physical and chemical stability of marine lipid-based liposomes under acid conditions. Colloids Surf. B Biointerfaces 20:257-266.

Nishizuka, Y. 1992. Intracellular signaling by hydrolysis of phospholipids and activation of protein kinase C. Science 258:607-614.

O'Connor, C. J., R. G. Wallace, K. Iwamoto, T. Taguchi, and J. Sunamoto. 1985. Bile salt damage of egg phosphatidylcholine liposomes. Biochim. Biophys. Acta 817:95-102.

Palmano, K. P., R. Ramos, M. Watson, K. E. Callon, and J. Cornish. 2011. Survival and bone-active properties of bovine lactoferrin supplemented into stirred yoghurt. Int. Dairy J. 21:477-483.

Roesch, R. R., A. Rincon, and M. Corredig. 2004. Emulsifying properties of fractions prepared from commercial buttermilk by microfiltration. J. Dairy Sci. 87:4080-4087.

Roseanu, A., P. E. Florian, M. Moisei, L. E. Sima, R. W. Evans, and M. Trif. 2010. Liposomalization of lactoferrin enhanced its antitumoral effects on melanoma cells. Biometals 23:485-492.

Satué-Gracia, M. T., E. N. Frankel, N. Rangavajhyala, and J. B. German. 2000. Lactoferrin in infant formulas: Effect on oxidation. J Agric. Food Chem. 48:4984-4990.

Sek, L., C. J. H. Porter, A. M. Kaukonen, and W. N. Charman. 2002 Evaluation of the in vitro digestion profiles of long and medium chain glycerides and the phase behaviour of their lipolytic products. J. Pharm. Pharmacol. 54:29-41.

Singh, H. 2006. The milk fat globule membrane-A biophysical system for food applications. Curr. Opin. Colloid Interface Sci. 11:154163.

Singh, H., and A. Sarkar. 2011. Behaviour of protein-stabilised emulsions under various physiological conditions. Adv. Colloid Interface Sci. $165: 47-57$.

Smith, A. M., M. R. Jaime-Fonseca, L. M. Grover, and S. Bakalis. 2010. Alginate-loaded liposomes can protect encapsulated alkaline phosphatase functionality when exposed to gastric $\mathrm{pH}$. J. Agric. Food Chem. 58:4719-4724.

Spitsberg, V. L. 2005. Invited review: Bovine milk fat globule membrane as a potential nutraceutical. J. Dairy Sci. 88:2289-2294.

Taylor, T. M., J. Weiss, P. M. Davidson, and B. D. Bruce. 2005. Liposomal nanocapsules in food science and agriculture. Crit. Rev. Food Sci. Nutr. 45:587-605.

Thompson, A. K., D. Haisman, and H. Singh. 2006a. Physical stability of liposomes prepared from milk fat globule membrane and soya phospholipids. J. Agric. Food Chem. 54:6390-6397.

Thompson, A. K., J. P. Hindmarsh, D. Haisman, T. Rades, and H. Singh. 2006b. Comparison of the structure and properties of liposomes prepared from milk fat globule membrane and soy phospholipids. J. Agric. Food Chem. 54:3704-3711.

Trif, M., C. Guillen, D. M. Vaughan, J. M. Telfer, J. M. Brewer, A. Roseanu, and J. H. Brock. 2001. Liposomes as possible carriers for lactoferrin in the local treatment of inflammatory diseases. Exp. Biol. Med. (Maywood) 226:559-564.

Trif, M., A. Roseanu, J. H. Brock, and J. M. Brewer. 2007. Designing lipid nanostructures for local delivery of biologically active macromolecules. J. Liposome Res. 17:237-248.

Troost, F. J., W. H. M. Saris, and R.-J. M. Brummer. 2002. Orally ingested human lactoferrin is digested and secreted in the upper gastrointestinal tract in vivo in women with ileostomies. J. Nutr. 132:2597-2600.

Troost, F. J., J. Steijns, W. H. M. Saris, and R.-J. M. Brummer. 2001. Gastric digestion of bovine lactoferrin in vivo in adults. J. Nutr. 131:2101-2104

Waninge, R., T. Nylander, M. Paulsson, and B. Bergenståhl. 2003. Milk membrane lipid vesicle structures studied with Cryo-TEM. Colloids Surf. B Biointerfaces 31:257-264.

Ye, A., J. Cui, and H. Singh. 2011. Proteolysis of milk fat globule membrane proteins during in vitro gastric digestion of milk. J. Dairy Sci. 94:2762-2770. 